\title{
Intervenções com pais: da alteração das práticas educativas parentais à inclusão de variáveis de contexto
}

\author{
Fabiane Ferraz Silveira \\ Universidade Federal de São Carlos
}

\begin{abstract}
Resumo
A justificativa para o treino de pais repousa na premissa de que os problemas de comportamento são produto das contingências de reforçamento dispostas pelos pais. O presente trabalho discute aspectos importantes de alguns programas de intervenções com pais, atribuindo maior ênfase às famílias que concomitantemente se deparam com problemas de comportamento dos filhos e enfrentam conflitos conjugais. Partindo deste posicionamento, apresenta-se um retrospecto de como os programas com pais evoluíram até o momento atual, variáveis de contexto e suas relações com problemas de comportamento e resultados de intervenções e pesquisas que versam sobre intervenção com pais, que incluem como objetivos a redução de problemas de comportamento associada à modificação do relacionamento conjugal; e discussão de limitações e avanços para estudos futuros.

Palavras-chave: problemas de comportamento; práticas educativas parentais; variáveis de contexto.
\end{abstract}

\begin{abstract}
Interventions with parents: the modification of parental practices and the inclusion of contextual variables. This paper aims to discuss aspects of some parent skills training program giving greater emphasis to families who concomitantly are faced with behavior problems and marital conflicts. From this view, discusses in the following section a retrospect, as the parent training program progressed until the present time; contextual variables and its relations with behavior problems and results of interventions; studies that include as objectives the reduction of behavior problems associated with the modification of conjugal relationship. In the end, the paper points out limitations and advances for further studies.
\end{abstract}

Keywords: behavior problems; parental practices; contextual variables.

$\mathrm{L}$ evantamentos realizados em diferentes Clínicas-Escola sinalizam a predominância de queixas de agressividade infantil nas entrevistas de triagem (Silvares, 2000). Dentre as alternativas propostas por pesquisas de caráter teórico ou com sustentação empírica, para o manejo da agressividade e demais tipologias de problemas de comportamento, pode-se destacar os programas de intervenções com pais. Este trabalho tem como objetivo, discutir aspectos importantes de alguns programas de treino de habilidades parentais desenvolvidos por pesquisadores nacionais e internacionais, com um destaque para as famílias que enfrentam problemas conjugais. Inicialmente será apresentado um retrospecto, de como os programas de treino de pais evoluíram até o momento atual. Posteriormente será discutida a influência das variáveis de contexto sobre os problemas de comportamento dos filhos e nos resultados de programas de intervenção com pais. Por fim, serão apresentadas pesquisas de intervenção com pais que estabelecem como objetivos, além da modificação da interação entre pais e filhos, também a inclusão de aspectos referentes ao relacionamento conjugal.

Problemas de comportamento podem ser diferenciados entre externalizantes, caracterizados por hiperatividade, impulsividade, oposição, agressividade e comportamentos internalizantes, tais como, disforia, retraimento, medo e ansiedade (Achenbach \& Edelbrock, 1979). O desenvolvimento de padrões externalizantes ainda na primeira infância, pode acarretar sérios prejuízos para a sociedade e para o indivíduo (Webster-Stratton \& Hammond, 1997).

A investigação dos determinantes dos problemas de comportamento incide sobre as inter-relações entre as características das crianças, dos cuidadores e do contexto social mais amplo (Ferreira \& Marturano, 2002). Os pais, ao desempenharem seu papel como agentes de socialização dos filhos, tentam orientá-los para o desenvolvimento da autonomia e responsabilidade e, para tal, utilizam estratégias denominadas de práticas educativas parentais (Alvarenga \& Piccinini, 2001). Práticas educativas parentais positivas como, por exemplo, disciplina consistente e supervisão favorecem a aquisição de comportamentos socialmente adequados (Gomide, 2004), ao passo que as negativas, que envolvem abuso físico e psicológico, negligência, ausência de atenção e de afeto estão associadas ao desenvolvimento de problemas de comportamento (BolsoniSilva \& Marturano, 2002). De acordo com Alvarenga e Piccinini (2001) as práticas educativas coercitivas são adotadas por pais e 
filhos como estratégia recorrente, pois operam em sobreposição uma contingência de reforçamento negativo para aquele que se submete ao controle, e uma contingência de reforçamento positivo, para o agente que dispõe de coerção.

Estudos indicaram uma correlação positiva entre problemas de comportamento e estratégias coercitivas e entre práticas educativas não coercitivas e comportamentos socialmente adequados (Fox, Platz, \& Bentley, 1995; Pettit \& Bates, 1989). Patterson, DeBaryshe e Ramsey (1989) propõem que a ocorrência de problemas de comportamento ainda na primeira infância, gera o afastamento dos pais, fracasso escolar e a aproximação de pares desviantes.

Segundo Webster-Stratton (1994), a justificativa para o treino de pais repousa na premissa de que os problemas de comportamento são produto das contingências de reforçamento dispostas pela família. Para Marinho (1999), a história de intervenção com pais tem relação direta com a história da modificação de comportamento. Após os experimentos de aprendizagem com animais e humanos e desenvolvimento de técnicas entre as décadas de 30 e 50, a modificação de comportamento incluiu o trabalho com pais como uma das vertentes aplicadas a partir da década de 60. Na década de 70, algumas limitações começaram a ser discutidas, principalmente relacionadas à questão da generalização dos resultados.

Segundo Silvares (1993), muitas das intervenções realizadas até a década de 80 basearam-se no modelo triádico. No modelo triádico, além do terapeuta e do cliente, os pais por disporem dos reforçadores em situação natural, atuam sob a orientação do clínico. Contudo, Wells (1983) salientou já na década de 80, a inadequação do modelo triádico para as crianças que não apresentavam dificuldades, contrariando as queixas dos pais, iniciando, a partir de então, a discussão da inclusão das variáveis de ajustamento da família no tocante à pesquisa e à intervenção.

Núcleos de pesquisas de intervenções com pais demonstraram resultados de generalização entre ambientes (Sanders \& Dadds, 1982), no tempo (Hartman, Stage, \& Webster-Stratton, 2003; Long, Forehand, Wierson, \& Morgan, 1994) e alta satisfação do consumidor (Webster-Stratton \& Hammond, 1997). Não obstante, pesquisadores nacionais - entre eles, Marinho (1999), Rocha e Brandão (1997) e Silva, Del Prette e Del Prette (2000), igualmente apresentaram resultados promissores. A última pesquisa citada avaliou a efetividade de um programa preventivo para pais, ou seja, cujos filhos não apresentavam indicativos de problemas de comportamento. Foram fortalecidas o que os autores denominam de habilidades sociais educativas como, por exemplo, iniciar e manter conversação, expressar afeto, controlar as próprias emoções e estabelecer regras. O programa mostrou-se efetivo em desenvolver habilidades essenciais para promoção de interações positivas entre pais e filhos.

Apesar de a extensa literatura atestar os efeitos positivos dos programas de treino de pais, críticos apontam que os discretos resultados obtidos com algumas famílias são explicados através do enfoque exclusivo nas práticas educativas parentais (Silvares, 2000; Webster-Stratton \& Hammond, 1997). BolsoniSilva, Villas-Boas, Romera e Silveira (2010) apresentam uma atualização do estado da arte de pesquisas sobre intervenção com pais. Foram analisados 192 resumos de artigos publicados entre os anos de 1986 e 2006, indexados nas bases de dados Index-Psi/Periódicos, LiLACS, PsycInfo, SciELO e Medline. Foi verificado que o atendimento em grupo é o mais comum, bem como o atendimento conjugado a pais e filhos. No tocante aos resultados, observou-se a redução dos comportamentos-problema como resultados principais e a promoção da competência social de pais e filhos como resultados adicionais pouco enfatizados.

Na mesma direção apontada por Bolsoni-Silva et al. (2010), algumas investigações ampliaram os atendimentos a adultos (pais e professores) e a crianças simultaneamente (Fernandes, Melo, \& Silvares, 2003; Webster-Stratton \& Hammond, 1997) e incluíram, além das práticas parentais, outras habilidades, tais como assertividade e resolução de problemas na avaliação e intervenção com pais, as quais estariam correlacionadas ao desenvolvimento comportamentos pró-sociais infantis (Baraldi \& Silvares, 2003).

Fernandes et al. (2003) avaliaram um treinamento de habilidades sociais com as crianças conjugado a um trabalho de orientação a pais e professoras. Participaram do estudo 38 crianças com, em média, oito anos, pertencentes a três grupos: a) 13 do grupo de intervenção conjugado pais e filhos; b) 12 do grupo controle (com indicação de dificuldades de interação, mas que não participaram de qualquer intervenção) e c) 13 do grupo de validação (sem indicação de dificuldades de interação). A avaliação sociométrica foi utilizada para mensurar a efetividade da intervenção. Foi solicitada antes, durante e após a intervenção, a cada companheiro de turma dos participantes do estudo (no total de 248 alunos), a indicação de três colegas com quem mais gostariam de brincar, três com quem menos gostariam e por quê. A intervenção com cada grupo de participantes (pais, crianças e professores) teve a duração de quatro meses. Os resultados sinalizaram uma alteração positiva no status sociométrico das crianças atendidas, comparadas às crianças sem atendimento, com destaque para as anteriormente classificadas como rejeitadas. Outra importante evidência da efetividade da intervenção refere-se à similaridade dos resultados pós-intervenção do grupo de atendimento e de validação social.

Webster-Stratton e Hammond (1997) compararam três modelos de intervenção: treino em grupo com crianças, treino em grupo com pais e um treino em grupo conjugado pais-criança. Participaram do estudo as famílias de 97 crianças de 4 a 8 anos com indicativos de problema de comportamento. A intervenção conjugada produziu melhores resultados quanto a problemas de comportamento, interações positivas entre pais e filhos e, principalmente, a manutenção dos resultados na avaliação de follow-up de um ano.

Baraldi e Silvares (2003) avaliaram a eficácia de uma intervenção conjugada a mães e filhos. Foi conduzido um treino de habilidades sociais e de resolução de problemas com 16 díades mãe-filho. O programa inicialmente programado para quinze sessões recebeu mais nove sessões adicionais, justificado por relatos de melhoras incipientes e baixa avaliação sociométrica das crianças. Os resultados dos filhos demonstraram uma diminuição de comportamentos de agressividade e aumento de obediência. Com relação às mães, foi observado o aumento de atenção e fornecimento de instruções. Outro dado relevante consistiu na observação de que quanto maior o número ou 
severidade das variáveis de contexto presentes - transtorno de humor e conflito conjugal - mais inferiores se situaram os desempenhos das mães.

A despeito das evidências de ganhos com a intervenção conjugada, especialmente, resultados de sucesso com todos os envolvidos (Fernandes et al., 2003) e manutenção dos resultados no follow-up (Webster-Stratton \& Hammond, 1997), inquestionáveis também são os indicativos da influência de variáveis de contexto nos padrões de interação familiares (Baraldi \& Silvares, 2003). Variáveis de contexto correspondem a fatores distais como cultura, escola, vizinhança; bem como proximais como desvantagem socioeconômica e conflito conjugal (Matos, 1983). As variáveis contextuais podem influenciar no desenvolvimento de problemas de comportamento, na medida em que alteram as práticas educativas parentais em perspectivas cronológicas distintas, isto é, atuando na primeira infância como resultado de desvantagem social ou posteriormente na história da família, em função de transtorno psiquiátrico dos pais ou stress (Patterson, Reid, \& Dishion, 1992).

Pesquisas sobre problemas de comportamento têm enfatizado os fatores de risco e proteção associados (Beauchaine, Webster-Stratton, \& Reid, 2005). Características da criança, tais como idade e temperamento e as variáveis de contexto, como vizinhança e desvantagem social, são considerados fatores de risco, ao passo que habilidades de manejo parental são entendidas como um importante fator de proteção (Forehand, Biggar, \& Kotchk, 1998). Na sequência são apresentados alguns estudos que investigam as variáveis de contexto, como fator de risco para o declínio das práticas educativas parentais e o desenvolvimento de problemas de comportamento.

\section{Variáveis de contexto: desvantagem social, vizinhan- ça violenta, depressão e conflito conjugal}

Segundo Ackerman, Brown e Izard (2004), uma constante dos estudos sobre populações economicamente desfavorecidas são os indicativos de que sequências ininterruptas de adversidades ambientais podem dificultar o desenvolvimento de habilidades de resolução de problemas e competência verbal. Exposições intermitentes a ambientes restritivos representam outro tipo de desafio às crianças, gerando sentimentos de baixa autoestima e de ausência de controle do ambiente (Ackerman et al., 2004).

Bank, Forgatch, Patterson e Fetrow (1993), por meio de um estudo longitudinal, verificaram que a trajetória direta da desvantagem social para a delinquência não foi significativa, oferecendo fortes bases para o modelo da mediação das práticas educativas parentais. Corroborando as hipóteses de Bank et al. (1993), Patterson et al.(1992) observaram em duas amostras distintas, famílias intactas e de mães solteiras, que a desvantagem social afeta de maneira mais significativa algumas práticas educativas, sobretudo disciplina consistente e controle emocional.

Muitas crianças experienciam ou observam manifestações de violência em casa ou na sua vizinhança. A exposição à violência pode estar associada à ansiedade, depressão e déficits de concentração (Margolin, Christensen, \& John, 1996).

Nos casos de violência presente na vizinhança, Margolin et al. (1996) afirmam que os esforços dos cuidadores podem se apresentar na forma de práticas educativas autoritárias e restritivas, influenciando, por seu turno, o aumento de respostas ansiosas na criança. De acordo com Forehand e Jones (2003) as práticas de monitoramento e estabelecimento de interações positivas garantem com que as crianças alcancem resultados expressivos em competência social e desempenho acadêmico, mesmo residindo em bairros violentos.

Margolin et al. (1996) verificaram que uma relação de confiança estabelecida com um adulto, assim como a avaliação positiva dos pares, representam fatores de proteção diante da vizinhança violenta. Atenções contínuas para os efeitos da violência sobre crianças nas diferentes fases de desenvolvimento, somado à caracterização dos fatores de risco e proteção, possibilitarão a identificação de novos objetivos para a intervenção e prevenção de problemas de comportamento.

Para Cummings, Keller e Davies (2005) a prevalência de depressão na população adulta e consequentes prejuízos nas relações familiares, profissionais e em situações sociais, sugerem também que um elevado número de crianças pode estar em risco para o desenvolvimento de problemas de comportamento. Pesquisas revelaram avaliações discrepantes de problemas de comportamento entre informantes, sendo que as mães com diagnóstico de depressão apontaram maiores ocorrências de comportamentos inadequados dos filhos se comparadas às avaliações das professoras (Brody \& Forehand, 1986; Treutler \& Epkins, 2003). Em uma perspectiva relacional, discriminações pouco precisas dos comportamentos adequados ou inadequados da criança podem produzir apresentações de consequências, com potencial efeito aversivo, não contingentes aos comportamentos da criança. Prerroga-se que mães deprimidas que passam pouco tempo com os filhos são mais irritáveis, apresentam dificuldades em prover uma rotina, e são mais propensas a agressões verbais e físicas, gerando pobre qualidade de interação tanto para as díades mãe-criança quanto para pai-criança (Cummings et al., 2005).

Depreende-se, a partir da literatura referenciada, que as variáveis de contexto estão associadas a diferentes dificuldades comportamentais, sobretudo, resolução de problemas, baixa autoestima e nas práticas parentais de controle emocional e disciplina consistente em se tratando da desvantagem social (Ackerman et al., 2004; Patterson et al., 1992); ansiedade, déficit de concentração e aumento nas práticas coercitivas no caso da vizinhança violenta (Forehand \& Jones, 2003; Margolin, 1996); e aumento na apresentação de punição não-contingente relacionado a quadros depressivos (Cummings et al., 2005). Cabe destacar o duplo papel das práticas educativas parentais na interação com as variáveis de contexto,: atuando como fator de proteção (Forehand et al., 1998) e na sobreposição de fatores de risco (Bank et al., 1993; Cummings et al., 2005).

As evidências de relações entre conflitos conjugais e problemas de comportamentos externalizantes e internalizantes são notórias (Cummings, Goeke-Morey, \& Papp 2004; Emery, 1982; Grych \& Fincham, 1990;). Emery (1982) propôs um construto para as consequências dos conflitos conjugais a partir de três componentes: a) o conflito conjugal é um evento aversivo que produz ansiedade na criança, b) o comportamento inadequado da criança produz uma alteração dos estímulos, que controlam as brigas dos pais, de questões do casal para 
questões relacionadas à criança e, c) aumento na probabilidade de ocorrência dos comportamentos-problema pela função que adquiriu para os pais e a criança, ou seja, a redução de um estímulo aversivo. Com esse processo de reforçamento negativo instalado, as crianças poderiam apresentar um padrão persistente e cada vez mais amplo de problemas de comportamento.

Em um estudo de revisão Cummings e Davies (2002) discutiram a distinção entre conflito conjugal destrutivo e construtivo, que envolvem, respectivamente, agressão física e verbal, ameaças de abandono do lar, discussões/agressões do casal relacionado à educação da criança; ouvir a opinião do outro e compromissos dos parceiros com a mudança. De acordo com Morey et al., (2007), os términos de conflito na forma de desculpas e compromisso com mudança produzem nas crianças reações emocionais negativas menos frequentes, se comparados aos términos de conflito com hostilidade verbal e isolamento.

A relevância dos processos responsáveis pelos efeitos indiretos e diretos do conflito conjugal nas interações familiares se traduz numa considerável agenda de propostas empíricas e conceituais (Buehler, Benson, \& Gerard, 2006; Kouros, Cummings, \& Davies, 2010; Schoupe-Sullivan, Schermerhorn, \& Cummings, 2007).

Buehler et al. (2006) avaliaram a influência das práticas parentais na relação entre hostilidade conjugal e problemas de comportamento em adolescentes. O estudo contou com 416 adolescentes e seus pais. Instrumentos padronizados de autorrelato foram utilizados como medidas de hostilidade, conflito conjugal, práticas parentais e problemas de comportamento. Provas estatísticas aferiram a significância das relações diretas ou indiretas das variáveis estudadas. A hipótese de mediação das práticas parentais na relação hostilidade conjugal e problemas de comportamento foi confirmada, sendo que a ausência de monitoramento e excesso de intromissões (intrusiveness) foram as variáveis de maior impacto.

Schoupe-Sullivan et al. (2007) investigaram as relações entre conflito conjugal, práticas parentais (controle, autonomia psicológica e afetividade) e comportamento externalizante e internalizante de crianças/adolescentes em 218 famílias. Como procedimentos de coleta de dados foram utilizados instrumentos padronizados de autorrelato. Os resultados indicaram que a relação entre conflito conjugal e problemas de comportamento foi mediada pelas práticas parentais, ou seja, foram encontrados efeitos indiretos do conflito conjugal sobre padrões externalizantes e internalizantes. Provas estatísticas revelaram que a relação conflito conjugal-comportamento externalizante é parcialmente explicada pelo decréscimo das práticas parentais; diferentemente do comportamento internalizante, cuja relação é totalmente explicada.

Kouros et al. (2010) examinaram o processo de influência do conflito conjugal sobre problemas de comportamento na infância e na competência social na adolescência, mediante um estudo longitudinal de cinco anos. Participaram da pesquisa 235 famílias e professores. Foram aplicados instrumentos padronizados de autorrelato com os pais, que avaliam conflito conjugal, comportamento externalizante na infância e repertório social na adolescência. Os professores também responderam ao instrumento de avaliação do repertório social na adolescência.
Correlações positivas significativas entre conflito conjugal e comportamento externalizante foram demonstradas, em outras palavras, o aumento do conflito conjugal prediz o aumento nos comportamentos externalizantes no decorrer do tempo. Foi verificado também que a ocorrência de comportamento externalizante na infância - sob a influência do conflito conjugal - está relacionada aos problemas de interação social na adolescência.

Observa-se que investimentos mais recentes apontam para modelos explicativos não estáticos, isto é, ao invés do estabelecimento de associações entre variáveis em um ponto específico do tempo, opta-se pela investigação da influência da variável conflito conjugal sobre os problemas de comportamento e repertório social no decorrer do tempo (Kouros et al., 2010), além de descrições mais acuradas do processo de mediação das práticas parentais (Buehler et al., 2006; Schoupe-Sullivan et al., 2007). Um dos desdobramentos possíveis, para as considerações apresentadas até o presente momento, faz referência ao fato de que providenciar aos pais intervenções focadas nas práticas educativas, se os mesmos relatam conflitos no relacionamento conjugal, poderá se mostrar inefetiva (Forehand \& Brody, 1985). Na sequência são discutidos aspectos concernentes às intervenções que abordam a coocorrência entre problemas de comportamento e conflito conjugal.

\section{Intervenções com duplo enfoque: problemas de com- portamento e conflito conjugal}

Apesar da demonstração empírica de relações entre conflito conjugal, práticas educativas ineficazes e problemas de comportamento, poucas pesquisas apresentam procedimentos de intervenção para a coocorrência de tais variáveis. Griest et al. (1982) compararam os efeitos de um programa básico de práticas parentais $(\mathrm{PB})$ e um programa extra $(\mathrm{PE})$, que além das práticas de manejo incluiu habilidades de comunicação e resolução de problemas. As avaliações pós-intervenção e follow-up, do grupo que recebeu o programa PE, revelaram uma expressiva redução na medida de problemas de comportamento se comparadas ao programa PB. As limitações do estudo destacadas pelos autores, dizem respeito à inexistência de diferentes medidas para problemas de comportamento e follow-up breve (três meses).

Webster-Stratton (1994) comparou os efeitos de dois programas: treino básico em práticas parentais e treino em práticas parentais com sessões extras em comunicação, resolução de problemas e habilidades de autocontrole. Os resultados da avaliação pós-intervenção indicaram que o grupo do treino extra apresentou resultados superiores em resolução de problemas e comunicação, porém resultados similares em problemas de comportamento contrariando as hipóteses iniciais. Na avaliação de follow-up não foram encontradas diferenças significativas nas medidas de satisfação conjugal e problemas de comportamento, novamente contrariando as hipóteses. Uma das explicações propostas considera que as mudanças favorecendo o grupo de sessões extras teriam um efeito atrasado, necessitando de mais tempo para gerar um impacto sobre as medidas comportamentais das crianças.

Sanders, Markie-Dadds, Tully e Bor (2000) compararam os resultados obtidos em três tipos de intervenção: o programa 
(EBFI), que integrava um treino de habilidades parentais com breve treino em comunicação e manejo de ansiedade, um programa padrão de treino em práticas parentais (SBFI) e uma variação deste programa (SDBFI) com orientações impressas para serem utilizadas pelos pais, sem contato com terapeutas. No pós-teste o programa (EBFI) não produziu resultados superiores aos outros programas em relação à redução de problemas de comportamento, práticas parentais e satisfação conjugal. No follow-up foram observadas diferenças significativas, favorecendo o EBFI, na medida de problemas de comportamento e resultados semelhantes entre os programas quanto a práticas parentais e satisfação conjugal. As análises sugeridas consideraram que os pais ao adquirirem certas habilidades de manejo de comportamentos inadequados dos filhos teriam menos oportunidades para discussão. Uma segunda possibilidade diz respeito à curta duração do treino de comunicação e manejo de ansiedade.

O estudo de Ireland, Sanders e Markie-Dadds (2003) teve como objetivo determinar se um programa com sessões extras em comunicação e resolução de problemas (EGTP) alcançaria resultados superiores se comparado ao programa padrão de habilidades parentais (SGTP). A hipótese era de que no pós-teste ambos os grupos teriam os mesmos resultados em relação às medidas de problemas de comportamentos dos filhos, práticas parentais e satisfação conjugal, entretanto, no follow-up, o EGTP estaria associado à melhores resultados. As hipóteses do pós-teste foram confirmadas, contudo, no follow-up as diferenças obtidas entre os grupos não atingiram níveis de significância. Algumas limitações apontadas pelos autores dizem respeito à inexistência de grupo controle, inexistência de medidas de observação, além de medidas de autorrelato e tempo restrito para as habilidades extras (cerca de 90 minutos).

A maioria dos estudos apresentados apontou para a similaridade de resultados entre programas que abordam somente práticas parentais e outros que incluem também treino de habilidades de comunicação, resolução de problemas e autocontrole (Ireland et al., 2003; Sanders et al., 2000; WebsterStratton, 1994). Contudo, algumas limitações metodológicas também foram destacadas em tais pesquisas, com possíveis relações com os resultados obtidos. Mesmo com as limitações metodológicas apontadas, alguns estudos verificaram melhoras em problemas de comportamento das crianças em algumas medidas desses comportamentos.

\section{Considerações finais}

Estudos internacionais sugerem a inexistência de um consenso quanto à extensão das mudanças em práticas parentais, problemas de comportamento da criança e satisfação conjugal ao incluir outras habilidades ao programa padrão. Observase que a inclusão das habilidades de comunicação, resolução de problemas e autocontrole não necessariamente reflete em mudanças na satisfação conjugal (Webster-Stratton, 1994), nem tampouco, em resultados superiores aos programas padrão no pós-teste, especificamente nas medidas de problema de comportamento e práticas educativas parentais (Sanders et al., 2000). Entretanto, no follow-up resultados superiores nas medidas de problema de comportamento, práticas educativas e satisfação conjugal, não suficientes de acordo com critérios estatísticos, são observados (Ireland et al., 2003).

Pesquisadores brasileiros ao desenvolverem modelos próprios de treinamento de pais, também sugerem a necessidade de considerações de outras variáveis atuantes sobre a família, dentre elas, o conflito conjugal (Baraldi, 2002; Silva et al., 2000; Silvares, 2000). Portanto, novas investigações com implicações teóricas e práticas precisam ser delineadas, uma vez que podem auxiliar na elaboração de intervenções preventivas.

Um dos desafios atuais para aqueles que se interessam pelo estudo das interações entre pais e filhos, produzem tecnologia comportamental, ou atuam em contexto clínico, é a consideração dos efeitos imediatos e/ou acumulativos das variáveis contextuais. Todas as famílias estão sujeitas a enfrentarem adversidades em diferentes momentos, podendo gerar consequências pífias em curto prazo. Outrossim, efeitos negativos acumulativos podem interferir no funcionamento familiar e, principalmente, no ajustamento psicossocial das crianças e jovens. Por fim, podemos concluir que um repertório vasto e bem estabelecido de práticas educativas parentais representa um importante fator de proteção para famílias sob a influência de múltiplas variáveis contextuais, e sobre o qual, todo investimento de profissionais e pesquisadores se justifica.

\section{Referências}

Achenbach, T. M., \& Edelbrock, C. S. (1979). Manual for the Child Behavior Checklist and revised child behavior profile. Burlington: University Associates in Psychiatry.

Ackerman, B. P., Brown, E. D., \& Izard, C. E. (2004). The relations between persistent poverty and contextual risk and children's behavior in elementary school. Developmental Psychology, 40(3), 367-377.

Alvarenga, P., \& Piccinini, C. (2001). Práticas educativas maternas e problemas de comportamento em pré-escolares. Psicologia: Reflexão e Crítica, 14(3), 449-460.

Bank, L., Forgatch, M. S., Patterson, G. R., \& Fetrow, R. A. (1993). Parenting practices of single mothers: mediators of negative contextual factors. Journal of Marriage and the Family, 55, 371-384.

Baraldi, D. M. (2002). Orientação a pais e ludoterapia comportamental com crianças em grupo (Dissertação de mestrado não publicada). Universidade de São Paulo, São Paulo.

Baraldi, D. M., \& Silvares, E. F. M. (2003). Treino de habilidades sociais em grupo com crianças agressivas associado à orientação dos pais: análise empírica de uma proposta de atendimento. In A. Del Prette \& Z. Del Prette (Orgs.), Habilidades Sociais, Desenvolvimento e Aprendizagem: questões conceituais, avaliação e intervenção (pp. 234-258). Petrópolis: Vozes.

Beauchaine, D., Webster-Stratton, C., \& Reid, J. M. (2005). Mediators, moderators, and predictors of 1-year outcomes among children treated for early-onset conduct problems: a latent growth curve analysis. Journal of Consulting and Clinical Psychology, 73(3), 371-388.

Bolsoni-Silva, A. T., \& Marturano, E. M. (2002). Práticas educativas e problemas de comportamento: uma análise à luz das habilidades sociais. Estudos de Psicologia, 7(2), 227-235.

Bolsoni-Silva, A. T., Villas-Boas, A. C. V. B., Romera, V. B., \& Silveira, F. F. (2010). Caracterização de programas de intervenção com crianças e/ou adolescentes. Arquivos Brasileiros de Psicologia, 62, 104-115.

Brody, G. H., \& Forehand, R. (1986). Maternal perceptions of child maladjustment as a function of the combined influence of child behavior and maternal 
depression. Journal of Consulting and Clinical Psychology, 54(2), 237-240.

Buehler, C., Benson, M., \& Gerard, J. M. (2006). Interparental hostility and early adolescent problem behavior: the mediating role of specific aspects of parenting. Journal of Research on Adolescence, 16(2), 265-292.

Cummings, E. M., \& Davies, P. T. (2002). Efects of marital confict on children: recent advances and emerging themes in process-oriented research. Journal of Child Psychology and Psychiatry, 43(1), 31-63.

Cummings, E. M., Goeke-Morey, M. C., \& Papp, L. M. (2004). Everyday marital conflict and child aggression. Journal of Abnormal Child Psychology, 32(2), 191-202.

Cummings, E. M., Keller, P. S., \& Davies, P. T. (2005). Towards a family process model of maternal and paternal depressive symptoms: exploring multiple relations with child and family functioning. Journal of Child Psychology and Psychiatry, 46(5), 479-489.

Emery, R. E. (1982). Interparental conflict and the children of discord and divorce. Psychology Bulletin, 92, 310-330.

Fernandes, R. E., Melo, M. H., \& Silvares, E. F. M. (2003). O julgamento de pares de crianças com dificuldades interativas após um modelo ampliado de intervenção. Psicologia: Reflexão e Crítica, 16(2), 309-318.

Ferreira, M. C. T., \& Marturano, E. M. (2002). Ambiente familiar e os problemas de comportamento apresentados por crianças com baixo desempenho escolar. Psicologia: Reflexão e Crítica, 15(1), 35-44.

Forehand, R., Biggar, H., \& Kotchk, B. A. (1998). Cumulative risk across family stressors: short-and long-term effects for adolescents. Journal of Abnormal Child Psychology, 26(2), 119-128.

Forehand, R., \& Jones, D. J. (2003). Neighborhood violence and coparent conflict: interactive influence on child psychosocial adjustment. Journal of Abnormal Child Psychology, 31(6), 591-604.

Fox, R. A., Platz, D. L., \& Bentley, K. S. (1995). Maternal factors related to parenting practices, developmental expectations, and perceptions of child behavior problems. The Journal of Genetic Psychology, 156, 431-441.

Gomide, P. (2004). Menor infrator: a caminho de um novo tempo. Curitiba: Juruá.

Griest, D. L., Forehand, R., Rogers, T., Briener, J. L., Furey, W., \& Williams, C. A. (1982). Effects of parent enhancement therapy on the treatment outcome and generalization of a parent training program. Behaviour Research and Therapy, 20, 429-436.

Grych, J. H., \& Fincham, F. D. (1990). Marital conflict and children's adjustment: a cognitive contextual framework. Psychological Bulletin, 108, 267-290.

Hartman, R., Stage, S. A., \& Webster Stratton, C. (2003). A growth curve analysis of parent training outcomes: examing the influence of child risk factors (inattention, impulsivity and hyperactivity problems) parental and family risk factor. Journal of Child Psychology and Psychiatry, 44(3), 388-398.

Ireland, J. L., Sanders, M. R., \& Markie-Dadds, C. (2003). The impact of parent training on marital functioning: a comparision of two group versions of the triple P-positive parenting program for parents of children wit early-onset conduct problems. Behavioral and Cognitive Psychotherapy, 31, 127-142.

Kouros, D. C., Cummings, E. M., \& Davies, P. T. (2010). Early trajectories of interparental conflict and externalizing problems as predictors of social competence in preadolescence. Development and Psychopathology, 22, 527-537.

Long, P., Forehand, R., Wierson, M., \& Morgan, A. (1994). Does parent training with young non-compliant children have long term effects? Behaviour
Research and Therapy, 32, 101-107.

Margolin, G., Christensen, A., \& John, R. S. (1996). The continuance and spillover of everyday tensions in distressed and nondistressed families. Journal of Family Psychology, 10, 304-321.

Marinho, M. L. (1999). Orientação de pais em grupo: intervenção sobre diferentes queixas comportamentais infantis (Tese de Doutorado não publicada). Universidade de São Paulo, São Paulo.

Matos, M. A. (1983). A medida do ambiente de desenvolvimento infantil. Psicologia, 9(1), 5-18.

Morey, M. C., Cummings, E. M., \& Papp, L. M. (2007). Children and marital conflict resolution: implications for emotional security and adjustment. Journal of Family Psychology, 21(4), 744-753.

Patterson, G. R., DeBaryshe, B. D., \& Ramsey, E. (1989). A developmental perspective on antisocial behavior. American Psychologist, 44(2), 329-335.

Patterson, G. R., Reid, J. B., \& Dishion, T. J. (1992). Antisocial boys. Eugene, Oregon: Catáslia.

Pettit, G. S., \& Bates, J. E. (1989). Family interaction patterns and children's behavior problems from infancy to 4 years. Developmental Psychology, 25, 413-420.

Rocha, M. M., \& Brandão, M. Z. S. (1997). A importância do auto conhecimento dos pais na análise e modificação de suas interações com os filhos. In M. Delitti (Org.), Sobre comportamento e cognição (Vol. 2, pp. 137-146). São Paulo: ARBytes.

Sanders, M. R., \& Dadds, M. R. (1982). The effects of planned activities and child management procedures in parent training: an analysis of setting generality. Behavior Therapy, 13, 452-461.

Sanders, M. R., Markie-Dadds, C., Tully, L. A., \& Bor, W. (2000). The triple P-Positive parenting program: a comparision of enhanced, standard and self-directed behavioral family intervention for parents of children with early onset conduct problems. Journal of Consulting and Clinical Psychology, 68(4), 624-640

Schoppe-Sullivan, S. J., Schermerhorn, A. C., \& Cummings, M. E. (2007). Marital Conflict and Children's Adjustment: Evaluation of the Parenting Process Model. Journal of Marriage and Family, 69, 1118-1134.

Silva, A. T. B., Del Prette, A., \& Del Prette, Z. A. P. (2000) Relacionamento paisfilhos: um programa de desenvolvimento interpessoal em grupo. Psicologia Escolar e Educacional, 3(3), 203-215.

Silvares, E. F. M. (1993). O modelo triádico no contexto da terapia comportamental com famílias. Psicologia: Teoria e Pesquisa, 11(3), 235-241.

Silvares, E. F. M. (2000). Terapia comportamental com famílias de crianças agressivas: porque, quando e como. Paidéia, 10(19), 24-33.

Treutler, C. M., \& Epkins, C. (2003). Are discrepancies among child, mother, and father reports on children's behavior related to parents' psychological symptoms and aspects of parent-child relationships. Journal of Abnormal Child Psychology, 31(1), 13-27.

Webster-Stratton, C. (1994). Advancing videotape parent training: a comparison study._Journal of Consulting and Clinical Psychology, 62(3), 583-593.

Webster-Stratton, C., \& Hammond, M. (1997). Treating children with early-onset conduct problems: a comparison of child and Parent Training Intervention. Journal of Consulting and Clinical Psychology, 65(1), 93-109.

Wells, K. C. (1981). Assesssment of children in outpatient settings. Em M. Hersen \& A. S. Bellack (Orgs). Behavioral assessment: a practical handbook (pp. 484-510). Nova Iorque: Pergamon Press.

Fabiane Ferraz Silveira, mestre em Psicologia do Desenvolvimento e Aprendizagem pela Universidade Estadual Paulista - Bauru, é doutoranda em Psicologia pela Universidade Federal de São Carlos. Endereço para correspondência: Rua Corifeu de Azevedo Marques, 3202/221C, Jardim das Indústrias, São José dos Campos-SP. CEP: 12241-040.

Telefones: (12)3322-4602/(12)9604- 4408. E-mail: fabianeferrazsilveira@yahoo.com.br 